\title{
REPRESENTACIONES CAMPESINAS SOBRE LOS SERVICIOS FORRAJEROS DEL CHACO SECO EN CÓRDOBA, ARGENTINA
}

\author{
SILVETTI, F. ${ }^{1}$
}

\section{RESUMEN}

El trabajo aborda las representaciones sociales de campesinos capricultores sobre los recursos forrajeros del bosque nativo en el Chaco Seco (Córdoba, Argentina). Se analiza la relación entre las condiciones sociohistóricas de acceso y uso del forraje y la construcción simbólica del ecosistema. Se concluye que los campesinos expresan una valoración territorial del conjunto de la oferta forrajera del bosque nativo, pero especialmente del denominado "monte bajo” (arbustal), principal recurso alimenticio para el ganado caprino. Estas representaciones guardan relación con el sistema de pastoreo extensivo que es la principal estrategia de apropiación campesina de los recursos forrajeros y se contraponen a las representaciones empresariales que sostienen la necesidad de erradicar el arbustal del territorio para implantar pasturas y aumentar la productividad del ganado bovino.

Palabras clave: campesinos, caprinos, representaciones sociales, servicios forrajeros, Chaco Seco.

\begin{abstract}
Social representations on forage resources offered by the dry Chaco native forest (Córdoba, Argentina).

The paper analyzes the capricultores social representations on forage resources offered by the Dry Chaco native forest (Córdoba, Argentina). The relationship between the historical conditions of access and use of forage by farmers and the symbolic construction of ecosystem is analyzed. It is concluded that farmers express a territorial assessment of the forage that provides the native
\end{abstract}

1.- Facultad de Ciencias Agropecuarias. Universidad Nacional de Córdoba. Avda. Valparaíso s/n, Ciudad Universitaria. (5000) Córodba, Argentina. Email: fsilveti@agro.unc.edu.ar

Manuscrito recibido el 27 de febrero de 2020 y aceptado para su publicación el 3 de junio de 2020.

F. Silvetti. Representaciones campesinas sobre los servicios forrajeros del Chaco Seco en Córdoba. FAVE Ciencias Agrarias 19 (1): 81-95. CC BY-NC-SA 4.0 (c) (1) (2) 
F. Silvetti

forest, but particularly that provides the "monte bajo" (scrub), main food source for goats. These representations related to the extensive grazing system is the main strategy of peasant use of forage resources of the territory. This view contrasts with the agrobusiness representatives that support the need to eradicate scrub to seeding new pastures and increase productivity of cattle.

Key words: peasants, goats, social representations, native forest, Dry Chaco.

\section{INTRODUCCIÓN}

El capitalismo manifiesta hoy una modalidad horizontal de penetración en los territorios rurales que reconfigura y/o excluye a los campesinos y sus modos de producir y reproducirse socialmente. La provincia de Córdoba (Argentina) constituye uno de los ejemplos más dramáticos en este sentido. El proceso de expansión del cultivo de soja y la intensificación ganadera bovina empresarial, ha generado un cambio en el uso de la tierra en el norte y oeste de la provincia de Córdoba, en detrimento de la superficie de bosque nativo y la provisión de servicios ambientales, entre los que se cuenta la disponibilidad de forraje (Cabido y Zak, 2010; Hoyos et al., 2013, Silvetti et al., 2018). Este esquema impone restricciones cada vez más severas al pastoreo extensivo de los rodeos caprinos, actividad que ha constituido durante siglos, la principal estrategia de sobrevivencia de los campesinos en el territorio (Silvetti, 2012). Desde la racionalidad de acumulación capitalista, se sostiene que el territorio constituye un espacio ambiental y socialmente problemático porque es productivamente "ineficiente” consecuencia del "atraso" y la "degradación ambiental” que produce y reproduce principalmente el sector campesino (LADA, 2004).

Esta visión dominante no considera la posición socialmente subordinada que los campesinos ocupan en relación a otros actores, tanto locales como extralocales, estructuralmente y políticamente mejor posicionados, cuya cuota de responsabilidad en la drástica disminución de la capacidad de los ecosistemas para proveer servicios esenciales para la vida ha sido y es sustancialmente mayor. Consideramos, por el contrario, que los sistemas campesinos han asegurado en gran medida la persistencia de las comunidades y la resiliencia de los ecosistemas (Silvetti, 2011). En este sentido Zak (2008), señala que en los departamentos del oeste de la provincia de Córdoba los ecosistemas mantienen una magnitud de servicios ecosistémicos importantes de preservar. Esto se debe a la permanencia en el territorio de sistemas campesinos dedicados principalmente a la cría de caprinos que necesitan de los servicios forrajeros del bosque nativo para poder sobrevivir ${ }^{1}$ (Silvetti, 2012).

1.- Los caprinos poseen, en relación a otras especies, mayor habilidad para convertir alimentos de baja calidad en productos de alto valor biológico como carne, leche, fibras, cueros y estiércol. Presentan gran flexibilidad en cuanto a la composición de su dieta, sobre todo en áreas áridas, donde consumen una mayor variedad de especies vegetales, seleccionan material vegetal de alta digestibilidad y tienen alta eficiencia para la digestión de forrajes de baja calidad (De Gea et al., 2005). 
En contraposición, el avance de la agricultura industrial en los departamentos del sur y centro norte de la provincia de Córdoba desde principios del siglo XXI, y el consecuente corrimiento de la ganadería a zonas más áridas, ha provocado un empobrecimiento irreversible de la biodiversidad de los ecosistemas provinciales, deteriorando las condiciones de persistencia de las comunidades campesinas (Silvetti et al., 2018). Los bosques pasaron, de cubrir el $44,1 \%$ del territorio provincial, a ocupar sólo el 7,7\%, y se estima que queda sólo el $5 \%$ de las 12.000 .000 hectáreas originales de bosque que poseía la provincia de Córdoba (COTBN, 2008).

En la región ganadera del noroeste de la provincia, el proceso de penetración capitalista se refleja en una fuerte concentración de las explotaciones dada por la desaparición creciente de los establecimientos de menos de 1.000 hectáreas (hasta un 51\%) y un aumento de los más grandes (hasta un $84 \%$ ). Asimismo, este último cambio refleja la ocurrencia de un proceso de "bovinización" de territorios históricamente abocados a la producción caprina. Efectivamente, el aumento de las existencias bovinas en el norte y oeste provincial es una consecuencia directa del desplazamiento de los sistemas de cría desde otros departamentos de la provincia, donde se ha extendido la agricultura o la ganadería se ha hecho más intensiva (Hocsman y Preda, 2005). Calvo et al. (2008), analizando la evolución del stock ganadero y el tipo de rodeos bovinos en Córdoba sobre la base de datos del Servicio Nacional de Sanidad Animal, señalan que en los departamentos del noroeste provincial, no sólo aumentó la relación novillito+novillo / vaca, sino que también se incrementó el stock ganadero bovino respecto del año 2002. En el caso del departamento Pocho, las existencias de ganado bovino se aumentaron un 89\% con relación a las cabezas que registra el CNA 2002 (Calvo et al., 2008). Cabe destacar que este proceso de "bovinización” está asociado a establecimientos agropecuarios con capacidad para financiarlas inversiones que requiere la intensificación productiva, tales como la construcción de alambrados perimetrales, instalaciones para reserva de agua y forrajes, y el reemplazo de la vegetación nativa por especies forrajeras exóticas. Es importante señalar que la intensificación de la producción ganadera que se observa en esta región contó con el apoyo del plan ganadero provincial "Más Terneros, Más Carne", financiado por la Secretaría de Agricultura, Ganadería, Pesca y Alimentos de la Nación, a través del CFI-PROSAP. Es importante señalar que la estrategia general de manejo de los establecimientos ganaderos empresariales, busca el reemplazo de la vegetación nativa, fundamentalmente arbustiva, por especies forrajeras megatérmicas a fin de aumentar la carga animal y la producción de carne. Esto implica, limitar las áreas de pastoreo tradicional que tenían los rodeos caprinos y eliminar el arbustal (llamado genéricamente como "fachinal") porque dificulta implantar pasturas y restringe la carga de bovinos. Para los productores ganaderos empresariales la valoración de los servicios forrajeros es "predial" y excluye a los "no propietarios". De esta manera se acelera la apropiación privada del forraje, que excluye a los campesinos, generando importante conflictos socioambientales (Silvetti et al., 2015).

El presente artículo analiza el manejo caprino campesino en el departamento Pocho, ubicado al oeste de la provincia de Córdoba, no sólo como una práctica social de apropiación material de los recursos forrajeros, sino también de apropiación sim- 
bólica, que se expresa en un sistema de representaciones sociales sobre la disponibilidad e importancia de los servicios forrajeros del Chaco Árido. Las representaciones sociales expresan la trayectoria histórica de uso de los servicios ambientales del territorio en función de los cambios que se sucedieron tanto a nivel del ecosistema como de las estrategias de reproducción social de los campesinos capricultores (Silvetti, 2012). En este sentido, Narotzky (2004:49) sostiene que el ecosistema es para los actores sociales una "experiencia vivida", además de ser el aspecto material que condiciona todo proceso de producción. Las condiciones biofísicas, que la sociedad interviene en la búsqueda de su sustento, se convierten en un resultado social e histórico, en el que las propiedades ecológicas y sociales se co-determinan.

La forma en que esta experiencia social de apropiación histórica del ecosistema se hace pública y comunicable es a través de las representaciones sociales. Para Jodelet (1986: 474), la representación social es "una forma de conocimiento específico, el saber de sentido común, cuyos contenidos manifiestan la operación de procesos generativos y funcionales socialmente caracterizados. Constituyen modalidades de pensamiento práctico orientados hacia la comunicación, la comprensión y el dominio del entorno material e ideal”. La representación tiene con su objeto una relación de simbolización, al ocupar su lugar, y de interpretación al conferirle significado. Estos significados resultan de una actividad de construcción y una expresión del sujeto, no sólo como individuo, sino como partícipe e integrante de una sociedad. Se trata de una construcción de la realidad psicológica y social a la vez (Jodelet, 1986).
Bourdieu (1997) sostiene que las representaciones sociales, además de generar un conocimiento de sentido común, poseen un contenido político al expresar los intereses asociados a las posiciones de los actores en un campo social y se convierten en un instrumento de la lucha por imponer determinadas visiones del mundo sobre otros. En consecuencia, abordar este saber de sentido común, diferente al académico o científico, tiene una importancia clave en tanto condiciona, los procesos psicológicos y cognitivos de los actores y el sentido de las interacciones sociales y con los ecosistemas.

\section{MATERIALES Y MÉTODOS}

El estudio acerca de las representaciones sociales campesinas acerca de los servicios forrajeros del Chaco Árido, forma parte de una investigación más amplia que analizó la relación entre las estrategias históricas de reproducción social de campesinos capricultores y la apropiación de los servicios ecosistémicos en el departamento Pocho, provincia de Córdoba (Silvetti, 2010). El trabajo de campo fue realizado en las pedanías Represa y Chancaní, hacia el oeste del mencionado departamento (Figura 1).

Las pedanías Represas y Chancaní constituyen una zona de llanura, incluida por Morello et al. (1985) en el distrito del Chaco Árido, que presenta precipitaciones menores a $600 \mathrm{~mm}$ con alto déficit hídrico (300-500 mm). Este distrito se compone originalmente de un estrato arbóreo superior, integrado por especies de alto valor económico y forestal que por su escasa densidad permite el desarrollo de un sotobosque compuesto por un estrato arbustivo 


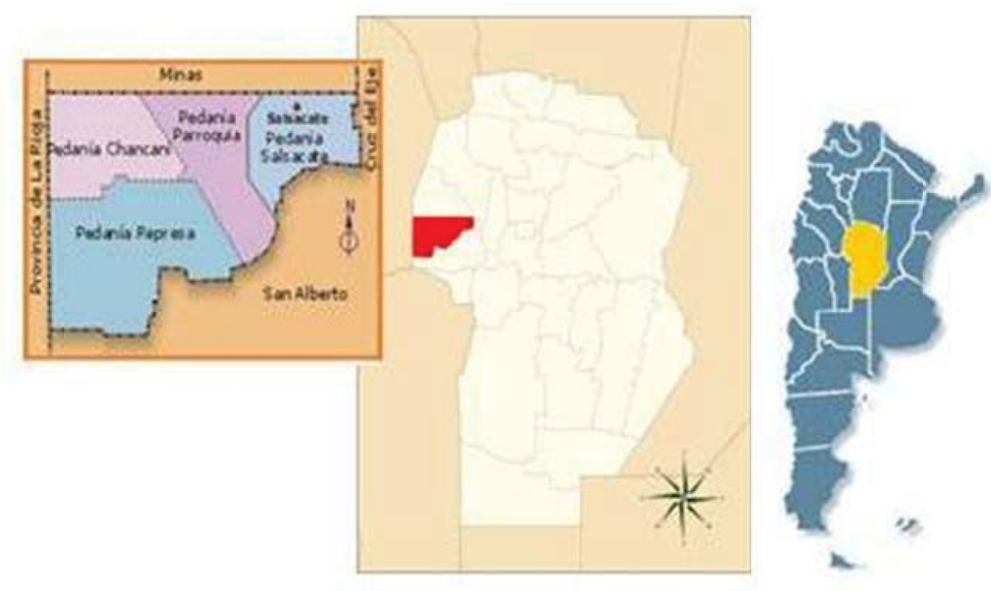

Figura 1. Ubicación del departamento Pocho en el oeste de la provincia de Córdoba (Argentina) Figure 1. Location of Pocho Department in Córdoba Province (Argentina)

y una cubierta inferior de gramíneas (Cabido et al., 1992). Debido al proceso de tala intensiva del estrato arbóreo que sufrió el territorio durante las primeras cuatro décadas del siglo XX, la superficie actualmente cubierta por el quebrachal de Apidosperma quebracho-blanco, se reduce a zonas protegidas en áreas de reserva (Reserva provincial Chancaní) y a territorios de piedemonte distal menos perturbados. En la llanura predominan los bosques bajos, los matorrales cerrados espinosos y los "peladares" que representan distintas fases de destrucción del bosque (Cabido et al., 1992). Según Zak (2008), el departamento Pocho muestra un predominio de bosques y matorrales de sustitución que ocupan más del 35\% de la superficie departamental.
Desde el punto de vista productivo, el área de estudio forma parte de la zona agroeconómica homogénea “Árida de traslasierras de ganadería extensiva”, donde el sistema productivo de relevancia es el minifundio ganadero con presencia de bovinos y caprinos (Ghida Daza y Sánchez, 2009). Según el CNA 2002, las pedanías Represa y Chancani cuentan con 158 establecimientos agropecuarios, que se pueden clasificar en 3 subtipos (Silvetti, 2010), cuyas principales características se presentan en la Tabla 1. Las unidades campesinas estudiadas están comprendidas en los subtipos "capricultores propietarios” y "capricultores ocupantes” (Silvetti, 2010). 
F. Silvetti

Tabla 1. Subtipos de establecimientos ganaderos identificados en las pedanías Represa y Chancani, departamento Pocho, provincia de Córdoba (Argentina)

Table 1. Subtypes of livestock producers, pedanías Represa and Chancani, Pocho department, Córdoba province (Argentina)

\begin{tabular}{|c|c|c|c|}
\hline $\begin{array}{l}\text { Indicadores } \\
\text { económicos }\end{array}$ & $\begin{array}{l}\text { GANADEROS } \\
\text { PROPIETARIOS }\end{array}$ & $\begin{array}{l}\text { CAPRICULTORES } \\
\text { PROPIETARIOS }\end{array}$ & $\begin{array}{l}\text { CAPRICULTORES } \\
\text { OCUPANTES }\end{array}$ \\
\hline $\begin{array}{l}\text { Porcentaje sobre el } \\
\text { total de EAPs }\end{array}$ & $17 \%$ & $37 \%$ & $46 \%$ \\
\hline Superficie promedio & 2.811 ha & 365 ha & 24,4 ha \\
\hline $\begin{array}{l}\text { Rodeo bovino } \\
\text { promedio }\end{array}$ & 297 cabezas & 50 cabezas & $\begin{array}{l}45 \% \text { tiene } 18,5 \text { cabezas. } \\
55 \% \text { no tiene bovinos. }\end{array}$ \\
\hline $\begin{array}{l}\text { Rodeo caprino } \\
\text { promedio }\end{array}$ & $\begin{array}{l}49 \% \text { posee } 109 \\
\text { cabezas. } \\
51 \% \text { no tiene } \\
\text { caprinos. } \\
\end{array}$ & 133 cabezas & 71 cabezas \\
\hline Rodeo ovino promedio & No poseen & No poseen & $\begin{array}{c}7 \% \text { tiene } 4 \text { cabezas. } 93 \% \text { no } \\
\text { tiene ovinos. }\end{array}$ \\
\hline Tenencia de la tierra & $\begin{array}{c}80 \% \text { son propietarios } \\
20 \% \text { sucesiones } \\
\text { indivisas }\end{array}$ & $\begin{array}{c}56 \% \text { propietarios } \\
37 \% \text { sucesiones } \\
\text { indivisas } \\
7 \% \text { ocupantes de tierras } \\
\text { privadas }\end{array}$ & $\begin{array}{c}32 \% \text { son ocupantes de } \\
\text { tierras comunales. } \\
24 \% \text { ocupantes de tierras } \\
\text { privadas } \\
22 \% \text { propietarios con límites } \\
\text { definidos } \\
22 \% \text { sucesiones indivisas } \\
\end{array}$ \\
\hline Vehículo & $90 \%$ posee & $25 \%$ posee & $98 \%$ no posee \\
\hline $\begin{array}{l}\text { Venta de fuerza de } \\
\text { trabajo }\end{array}$ & No & No & $10 \%$ vende \\
\hline $\begin{array}{l}\text { Promedio de personas } \\
\text { residentes en la EAP }\end{array}$ & 2 personas & 4 personas & 6 personas \\
\hline
\end{tabular}

La investigación fue enfocada como un estudio de caso (Valles 1997) bajo los criterios metodológicos de la triangulación de métodos que guía la investigación socioantropológica etnográfica (Combessie, 2005: 16). Para el relevamiento de la información se realizaron entrevistas en profundidad a unidades domésticas campesinas y otros informantes clave, encuestas semi-estructuradas para relevar información cuanti-cua- litativa referida a los sistemas campesinos, observación participante en las visitas a los predios y consulta a fuentes primarias y secundarias. El criterio acerca del universo de unidades domésticas a considerar, se basó en una muestra intencional, donde la selección de los casos está orientada al desarrollo de "teoría fundamentada" (Glaser y Strauss, 1967). 
La información específica referida a las representaciones campesinas sobre los servicios forrajeros fue obtenida a través de entrevistas en profundidad a 42 unidades domésticas campesinas ${ }^{2}$ que fueron grabadas y transcriptas. La información de las entrevistas fue codificada según categorías descriptivas y conceptuales emergentes (Strauss y Corbin, 1990). Con el material reunido en cada sección se realizó una integración local de la información que resultó en las categorías de clasificación y valoración campesino de los servicios forrajeros del territorio. La interpretación se centró en el material discursivo acumulado en cada una de las categorías emergentes (Weiss, 1995).

\section{RESULTADOS Y DISCUSIÓN}

Según Silvetti (2012) desde el siglo XVI hasta la actualidad, el departamento Pocho ha transitado por un proceso de construcción social del hábitat que resulta en tres tipos de territorialidades ${ }^{3}$ : i) la territorialidad campesina ganadera, originada en la época colonial y orientada a la producción y consumo local de caprinos en base al pastoreo extensivo del forraje provisto por el bosque nativo; ii) la territorialidad extractivista, a partir del avance del capitalismo en el territorio, con el advenimiento del ferrocarril a principios del siglo XX, la explotación de los recursos forestales, la proletarización de la mano de obra campesina y la mercantilización de los productos de las economías domésticas campesina y; iii) la territorialidad empresarial ganadera, con la intensificación de la ganadería bovina a partir de la primera década del siglo XXI y la concentración de la tierra en detrimento de los intereses y actividades productivas de los campesinos, tal como se explicitó en la introducción. En consecuencia, los efectos combinados de la deforestación desde principios del siglo XX y la intensificación agropecuaria empresarial en el siglo XXI, han afectado la biodiversidad del bosque chaqueño y resentido la provisión de servicios ecosistémicos de los cuales dependen las unidades domésticas campesinas para sobrevivir.

Frente a la valoración y uso predial de los servicios forrajeros del territorio que promueven los ganaderos bovinos empresariales, las representaciones campesinas acerca del uso y valor de los servicios forrajeros del bosque nativo responden a un es-

2.- La unidad doméstica puede ser conceptualizada como una organización social que tiene como objetivo la realización de procesos de producción, distribución y consumo necesarios para el mantenimiento y reproducción social de sus integrantes corresidentes, por lo que el vínculo entre los miembros no es exclusivamente el parentesco (Cragnolino, 1997). Es necesario que prevalezca en la unidad doméstica un espíritu de cuerpo (Jelin, 1984; Bourdieu 1990) a los fines de poder llevar adelante las actividades cotidianas, que se logra a través de un trabajo de inculcación social y legitimación simbólica del esfuerzo diferencial que se demanda a cada individuo (según género y edad por ejemplo) en beneficio de un colectivo, determinando posiciones de poder diferenciales para organizar la producción, la distribución y el consumo (Narotzky, 2004).

3.- Es importante señalar muchos rasgos de estas tres territorialidades aún permanecen y coexisten en el territorio a pesar de las transformaciones sufridas. 
quema histórico de pastoreo extensivo que sobrepasa los límites prediales, permitiendo a las unidades campesinas utilizar grandes áreas de pastoreo en forma comunitaria, con independencia del número de cabezas y/o la superficie de tierra que cada unidad doméstica dice poseer. Durante muchas décadas, existieron arreglos locales informales entre los capricultores y los productores de ganado bovino tradicionales que regulaban el pastoreo de los rodeos caprinos en sus predios (Silvetti, 2012). En consecuencia, la valoración de los recursos alimenticios para el ganado que realizan los campesinos se refiere a la superficie real que pastorean los caprinos en el territorio y es por ello que las categorías de forrajes identificadas por los campesinos aluden a una heterogeneidad de configuraciones vegetales.

Los campesinos consideran diferentes condiciones del monte para construir un sistema de clasificación de los servicios forrajeros vinculados a la alimentación del ganado caprino. Los servicios forrajeros que provee cada categoría son valorados según un conjunto de atributos tales como: valor nutritivo, diversidad estacional, aporte de biomasa estacional, abundancia relativa de ciertas especies vegetales y palatabilidad. También se incluyen algunos aspectos negativos que derivan de la presencia de sustancias tóxicas para el ganado o de la dominancia de especies espinosas que dificultan el paso de los animales o los lastiman.

La accesibilidad que presenta el forraje según la altura de ramoneo de los caprinos "la cabra come hasta donde alcanza" (aproximadamente $1,50 \mathrm{~cm}$ ), es el principal aspecto que los campesinos tienen en cuenta para clasificar los servicios forrajeros del bosque nativo. Estos tipos son: monte alto, monte bajo y pastos. Cada categoría pre- senta características específicas según su aporte a la nutrición del ganado que desarrollamos a continuación:

a) La categoría "monte alto" hace referencia al estrato arbóreo del bosque nativo. Las especies incluidas por su valor nutricional son: algarrobo negro (Prosopis flexuosa), algarrobo blanco (Prosopis alba), tala (Celtis tala), mistol (Ziziphus mistol) y chañar (Geoffrea decorticans). El alimento que los animales obtienen del monte alto son los productos que "caen de los árboles" tales como frutos y hojas senescentes. Es importante destacar que debido a que los renovales de árboles presentan frutos, hojas y ramas tiernas accesibles al ramoneo del ganado, son incluidas como parte de la categoría "monte bajo". Si bien todos los frutos de los árboles constituyen para los campesinos un aporte apreciable para el ganado (e.g. chañar y mistol), se reconoce en la "algarroba" (fruto de los algarrobos) un valor nutricional excepcional que queda evidenciado en los efectos positivos que pueden observar en los animales, "la algarroba es el manjar de las cabras". Los frutos del algarrobo se producen en el verano, desde fines de diciembre a febrero. Maduran en la planta y cuando caen son consumidos desde el suelo por los animales. En años donde hay suficiente algarroba disponible en época estival, el ganado se recupera rápidamente de la desnutrición invernal, aumenta la producción de leche en cabras, disminuye la incidencia de enfermedades, los animales aumentan de peso, están más fuertes: "los animales se ponen lindos"; "la gordura les dura”. Asimismo, el consumo de algarroba durante el verano, prepara al ganado para llegar a la época invernal en mejores condiciones nutricionales. 
Antes de que la explotación forestal disminuyera considerablemente la disponibilidad forrajera del monte alto, las familias cosechaban los frutos de los árboles para ser guardados como reserva alimenticia para la época invernal (Silvetti, 2010). Actualmente, sólo en años de excepcional abundancia de frutos, existe un remanente posible de ser recolectado. Esta práctica social tiene un valor muy importante en un ecosistema con una concentración estacional estival de la productividad primaria de la vegetación, en un territorio sin posibilidades de implementar un sistema de trashumancia de los caprinos, tal como se hace en otras regiones del país. Pero esta práctica fue abandonada progresivamente debido a la disminución del número de árboles por la explotación forestal comercial desde principios del siglo XX, los frecuentes incendios y el deterioro de ejemplares por la difusión de plagas de insectos xilófagos como el taladrillo y otras enfermedades: "Los algarrobos se apestaron con el taladrillo que se mete en el tronco o en la raíz y los empieza a secar. Atrás del taladrillo viene siempre la hormiga, hacen hormigueros adentro y el árbol se seca. No sirven ni para leña. Antes el algarrobo era muy importante y también había mucho quebracho. Menos mal que la gente les sacó provecho con la leña y el carbón. Para la hacienda es más fiero en invierno porque le falta reparo.” (entrevista M.G., El Medanito).

La disminución de la disponibilidad de la mano de obra familiar necesaria para realizar la recolección estacional de los frutos, debido a la emigración sostenida de la población que ha sufrido el departamento Pocho, también ha tenido incidencia en el abandono de la costumbre de recolectar los frutos de los árboles nativos (Silvetti, 2012).
La producción estival de frutos está condicionada por múltiples factores tales como, el número de ejemplares, la edad de los árboles, la incidencia positiva o negativa de los elementos del clima sobre la floración y fructificación, las enfermedades y plagas y las condiciones de fertilidad del suelo. Si bien los campesinos coinciden en que los algarrobos son en general muy productivos, consideran que los ejemplares más jóvenes son los que generan más vainas por árbol: “Cuando el monte es más nuevo da más algarroba. Cuando el monte es más viejo, menos fruta da. En casa hay unos algarrobos de más de 100 años y da poca fruta, en cambio los algarrobos de 7 años dan más algarroba. Un algarrobo en 2 o 3 años da frutos. La algarroba se usaba para engordar a todos." (entrevista R.M., Santa Rosa).

La masa foliar del monte alto es identificada por los productores como un aporte alimenticio de relevancia en el momento de senescencia: "las cabras comen el hojaraje”. Este aporte de hojas muertas comienza con las primeras heladas y se mantiene según las especies hasta fines del invierno. Esto también constituye una ventaja desde la perspectiva de los capricultores porque se escalona temporalmente el aprovechamiento del recurso. En el caso de los algarrobos, producen una pérdida de hojas al rebrotar hacia el final del invierno y el comienzo de la primavera, momento crítico en que la oferta forrajera general del ecosistema es la más baja del año, por lo que este recurso es especialmente apreciado por los campesinos. El tala en cambio, pierde la hoja cuando comienza el período de heladas, desde mediados de mayo y comienzos del invierno y es valorado porque es una especie relativamente abundante debido a que 
F. Silvetti

no ha sido explotada comercialmente por su escaso valor maderable y calorífico. En cuanto a la palatabilidad que presentan las diferentes especies, los productores se refieren a que los caprinos prefieren las hojas dulces y jugosas (de los algarrobos) a otras especies que son más amargas o secas. Sin embargo, en condiciones de mucha humedad, tanto los frutos como las hojas que se encuentran en contacto con el suelo, se descomponen rápidamente o se pierden por el accionar de insectos u hongos y a los pocos días han desaparecido: "con el temporal las hojas duran poco", "los frutos se aguanacan [se pudren]".

El efecto benéfico que genera la sombra de los árboles sobre las especies vegetales que crecen bajo el dosel y sobre el ganado es para los campesinos un aspecto importante de considerar. Los animales se benefician por la protección física que ofrece la canopia del bosque y la regulación de la temperatura que genera:"Es bueno que el campo tenga sombra, por ejemplo ahora, que ya han vuelto los animales de comer, se han echado a la sombra del árbol [algarrobo] y del mistol, que tienen sombra muy buena, y ahí se ponen a reposar. Hay quienes dicen que la sombra no deja que salga pasto. Pero yo diría que puede ser verdad, pero que en verano, cuando están los soles tan fuertes, ahí la sombra favorece para que salga pasto, porque no lo quema tanto. $Y$ sino, a los pastos que están como ahí en la playa, el sol los quema en seguida. No, yo creo que es buena la sombra..." (entrevista a R.C, Chancaní).

Para la mayoría de los campesinos entrevistados, la tala del estrato arbóreo significó un aumento inmediato de la productividad de los estratos inferiores, tanto de especies arbustivas como herbáceas al poder captar más luz y porque hay un aporte adicional de materia orgánica al suelo: "al caer los restos del monte es como que el suelo se abonara". Sin embargo, algunos observan un efecto negativo vinculado con la mayor pérdida de agua del suelo en las zonas donde queda desnudo y expuesto en forma directa a la radiación solar. Debido a que en la zona, el recurso más crítico es el agua antes que la luz, vemos que cuando los productores se refieren específicamente a los factores que inciden sobre la productividad de las pasturas en particular, el agua es el elemento colocado en primer lugar: “...donde hay más monte, cuando llueve dura mucho más la humedad, donde está pelado ahí nomás se seca. En las playas, el pasto es como que no sale, no hay pasto, donde no hay monte no ha salido casi pasto. Yo creo que el mismo monte hace falta también para que el pasto crezca, pero muy tupido no, porque les interrumpe a las vacas para caminar." (entrevista R.B, El Cadillo).

b) La categoría "Monte bajo" incluye al arbustal que actualmente cubre el 35\% del territorio (Zak, 2008) y el renoval de los árboles. Las especies arbustivas incluidas son muy numerosas: tusca (Acacia aroma), espinillo (Acacia caven), mistol del zorro (Castela coccinea), garabato (Acacia praecox), tintitaco (Prosopis torquata), atamisqui (Cappans atamisquea), piquillin (Condalia microphylla), sombra de toro (Jodina rhombifolia), tala churqui (Celtis pallida), abriboca (Maytenus spinosa), poleo (Lippia turbinata), teatín o garabato negro (Acacia fucartispina), garabato hembra (Mimosa detinens), jarilla ( $L a-$ rrea divaricata), pichana (Semma aphylla), cachiyuyo o zampa (Atriplex lampa). Dentro de este monte bajo se consideran también las cactáceas (e.g. cardones, ucles y quimilos) pero con un valor forrajero muy secundario. 
El monte bajo es caracterizado como "monte tupido, denso, con mucho churqui, joven, cubierto, bravo, espinoso, de rehache”. Provee actualmente la mayor biomasa y biodiversidad disponible para la alimentación de los rodeos caprinos. Ello se debe al proceso de arbustización que se sucedió a la extracción del estrato arbóreo en el territorio con motivo de la tala intensiva con fines comerciales. Las especies arbustivas y el renoval son especialmente valorados por los campesinos debido a su relativa abundancia, estacionalidad, diversidad y accesibilidad para los animales. Debido a que la altura de las especies no excede la altura de ramoneo de los caprinos, el animal puede consumir en forma directa la totalidad de la biomasa disponible: hojas, flores, frutos y ramas tiernas. El valor nutricional de las vainas de lata, tusca y garabato son especialmente valoradas por los campesinos:“Un lindo campo para las cabras es donde hay mucho churqui, el tala petizo, un tala que se cría bajo, y es muy lindo para la cabra. También come mucho el poleo. Pero el bueno es el tala petizo, porque hay mucho tala grande también, es el que más les gusta. Cuando cae la helada y las hojas que están alto caen, se le van al humo a esas hojitas [...] La cabra principalmente ramonea, o se come las hojas del suelo. El mistol les gusta mucho a las cabras. El tala, el algarrobo, el churqui es lo que más le gusta a la cabra" (entrevista A.G., Chancaní).

El aprovechamiento por parte de los animales del monte bajo se escalona según las distintas épocas del año y complementa el aporte más concentrado en frutos y hojas que realiza el monte alto. La mayor oferta forrajera se concentra en el período primavera-estival y disminuye al finalizar el período invernal y comienzo de la prima- vera. Ello obliga al ganado a ir ampliando progresivamente la superficie territorial de pastoreo con los consiguientes problemas de pérdidas, robos y predación de animales. En la época más crítica del bache forrajero, al comienzo de la primavera, son apreciadas las especies de rebrote temprano como el mistol del zorro o especies perennes como el cachiyuyo, que se adaptan mejor a suelos más pobres, salitrosos o medanosos.

Para los campesinos la accesibilidad de las cabras al recurso forrajero del monte bajo se ve dificultada cuando presenta una estructura “cerrado y espinoso", llamado "fachinal": "Un fachinal es un campo digamos inútil, que está todo cubierto de churqui, de monte con espina, donde no entra nadie, solo las fieras chiquitas, los bichos chiquitos, las víboras...” (entrevista R.B, El Cadillo).

Esta configuración impide que las cabras puedan transitar con comodidad y favorece que sus patas queden atrapadas entre las ramas espinosas cuando intentan alcanzar las hojas más altas de los arbustos, generando heridas de consideración y hasta la muerte. En este caso los productores prefieren que el monte esté más abierto, aunque reconocen que son los mismos caprinos los que "van abriendo el monte" efecto que no sucede con el ganado bovino.

El monte bajo también fue afectado por la explotación forestal porque muchas especies sirvieron para elaborar "carbón mezcla” o para obtener postes y varillas. Las áreas de bosque especialmente empobrecidas se identifican por la dominancia de la jarilla, a la cual los campesinos le atribuyen escaso valor alimenticio y es además evaluada como una de las condiciones menos deseadas en términos de provisión de forraje. Incluso reconocen que son un indicador 
F. Silvetti

de áreas sobrepastoreadas. Como ya se señaló en la introducción, es precisamente el "monte bajo" la configuración más amenazada en el territorio por el proceso de bovinización empresarial, ya que los ganaderos la visualizan como un obstáculo para implantar pasturas que aumenten la oferta forrajera en sus predios.

c) La categoría “pastos” corresponde a las especies herbáceas nativas del Chaco Seco (e.g. Goguinia spp., Triclhoris spp., Pappophorum spp., Setaria spp, Bouteloa aristidoides). Lo llamativo para esta categoría es que, a diferencia de las dos categorías anteriores, los productores realizan una evaluación más a escala predial que territorial sobre su disponibilidad y productividad. Esto se debe a que el pasto es considerado un forraje principalmente orientado a la alimentación del ganado bovino más que al caprino. Refleja además una tendencia en las unidades domésticas campesinas con escasez de mano de obra familiar, que buscan reemplazar los caprinos por bovinos para simplificar el manejo, ya que el manejo caprino es intensivo en mano de obra (Silvetti, 2012).

Dentro de la variedad de pastos presentes, el más valorado por su valor alimenticio es el llamado "pasto raíz" (Trichloris spp.) por tratarse de un pasto plurianual, de rebrote temprano, especialmente importante para la alimentación de los animales. A diferencia de los bovinos que prefieren pasturas blandas y poco amargas, las cabras pueden aprovechar los pastos que son más amargos y duros (De Gea et al., 2005). El pastoreo conjunto de caprinos y bovinos es considerado por algunos, como una situación complicada porque los ganaderos argumentan que el pisoteo y las deyecciones de los caprinos tienen efectos negativos sobre la calidad de las pasturas. Debido a que estos argumentos pueden utilizarse para limitar el pastoreo de las cabras en predios predominantemente ganaderos, los capricultores enfatizan la escasa preferencia que tienen los caprinos por consumir las herbáceas: “Todas las cabras están juntas en lo de $R$. porque el viejo ha rodeado [con alambrado] todo, porque tiene campos por todos lados. El año pasado se puso jodido. Si llega a vender y viene algún delicado del sur no se qué va a hacer la gente de acá de la zona, porque han rodeado todo Santa Rosa, El Quemado, La Patria, todo han rodeado....Esta zona de Chancaní y Santa Rosa, todas esta parte, todas las familias tienen cabras. Es el único medio de vida... Gracias a Dios todavía no hemos tenido problemas con $R$. Hay miles de animales en su campo pero como le digo yo las cabras no comen el pasto, les gusta el monte. Pero por ahí el hombre conversa con los otros ricachones y empieza que le orinan el pasto, que le pisotean y estamos listos!” (entrevista E.Q., El Quemado). Como vemos, para los capricultores, es estratégico sostener un discurso en términos de aprovechamiento diferencial de los estratos vegetales por parte de bovinos y caprinos a fin de minimizar conflictos con productores ganaderos extralocales, que como recién llegados, no sólo desconocen los acuerdos históricos que existían con los ganaderos tradicionales, sino que tienen el poder para restringir las áreas de pastoreo, a través del alambrado de los campos y la sustitución del monte por pasturas implantadas. 


\section{CONCLUSIONES}

La apropiación social de determinados recursos forrajeros del bosque nativo no sólo implica analizar las condiciones sociales de acceso material y gestión de los mismos en el marco de las estrategias históricas de supervivencia campesinas, sino también las representaciones sociales vinculadas, que comunican sistemas de clasificación y valoración de los recursos y un posicionamiento de los campesinos frente a los procesos territoriales contrarios a sus intereses.

Los campesinos expresan una valoración territorial de la oferta forrajera que provee el conjunto de los estratos vegetales del bosque nativo, pero en particular los que brinda la vegetación encuadrada en la categoría campesina "monte bajo" (arbustal), actualmente predominante en el territorio y principal recurso alimenticio para el ganado caprino. Estas representaciones guardan relación con el sistema de pastoreo extensivo que es la principal estrategia de apropiación campesina de los recursos forrajeros del territorio. Pero es la biodiversidad del bosque nativo lo que permite organizar un sistema de pastoreo extensivo que excede los límites prediales, con un aprovechamiento horizontal y vertical de los estratos vegetales y escalonados en el tiempo. Esta mirada se contrapone a las representaciones ganaderas empresariales que sostienen la necesidad de erradicar el arbustal del territorio para implantar pasturas y aumentar la productividad del ganado bovino. Al respecto Díaz (2010), ecóloga especialista de CONICET, argumenta en contra de esta mirada ante la Comisión de Asuntos Ecológicos de la legislatura cordobesa: "El 60 por ciento del carbono [de los bosques nativos] de Córdoba está en el bosque secundario y arbustales mixtos, mal llamado fachinal.... Los fachinales proveen de servicios ecológicos a productores y a toda la comunidad. No son improductivos como se piensa. Los productores de subsistencia viven de ellos, tienen ganadería, miel y combustible. Para la sociedad son un recurso genético, tienen un potencial turístico y sirven como corredores para las especies que viven en las reservas".

En el siglo XXI, las modalidades usuales de apropiación de los recursos son amenazadas por los nuevos procesos que comienzan a delinearse en el territorio: la competencia con la ganadería bovina y la mayor fiscalización por parte del Estado sobre la explotación forestal del bosque nativo (Silvetti et al., 2013). El nivel de provisión actual de los servicios forrajeros no sólo está comprometido por el efecto de la tala intensiva que empobreció el ecosistema original, sino por una revalorización económica de la tierra y una subvaluación del arbustal que produjo un cuestionamiento de los acuerdos sociales históricos que sostenían el pastoreo extensivo como el sistema dominante en el territorio. Si bien durante décadas se había generado una organización socioespacial para vivir del monte por parte de los campesinos, en las primeras décadas del siglo XXI, el proceso de bovinización empresarial plantea un conflicto de intereses múltiple entre los actores por el acceso a los servicios forrajeros y su uso. Ello pone en peligro las posibilidades de los campesinos para acceder y apropiarse de los servicios forrajeros del territorio, erosionando las bases de su reproducción social. 


\section{BIBLIOGRAFÍA}

BOURDIEU, P. 1990. La domination masculine. Actes de la Recherche en Sciences Sociales, 84 (9): 2-32.

BOURDIEU, P. 1997. Razones prácticas. Sobre la teoría de la acción. Barcelona: Anagrama. $232 \mathrm{pp}$.

CABIDO, M.; ZAK, M. 2010. Deforestación, agricultura y biodiversidad: Apuntes sobre el panorama global y la realidad de Córdoba. Revista HOY la Universidad - UNCiencia. Universidad Nacional de Córdoba. Córdoba. Disponible: http://m.unciencia.unc. edu.ar/2010/junio/deforestacion-agricultura-y-biodiversidad-apuntes

CALVO, S.; SALVADOR, M.L.; GONZÁLEZ PALAU, C.; IGLESIAS, D. 2008. Anexo 19: La cadena de carne bovina en la provincia de Córdoba. Implicancias para el desarrollo regional. Instituto de Investigaciones Económicas. En El balance de la economía Argentina 2008. Córdoba: Bolsa de Comercio de Córdoba IIE. pp. 1-28.

CNA. 2002. Censo Nacional Agropecuario. Buenos Aires: Instituto Nacional de Estadísticas y Censos.

COMBESSIE, J.C. 2005. El Método en Sociología. Córdoba: Ferreyra Editor. 174 pp.

COMISIÓN DE ORDENAMIENTO TERRITORIAL DEL BOSQUE NATIVO (COTBN). 2008. Proceso y programa de la OTBN de la provincia de Córdoba. Propuesta de ley de ordenamiento territorial de bosques nativos. Disponible: http://cotbn2009. files.wordpress.com/2009/06/ley-otbn.pdf.

DÍAZ, S. 2010. Los fachinales conservan el $60 \%$ del carbono de los bosques de Córdoba. La Voz del Interior. Ambiente. Consultado el 30 de marzo 2010 en http://www. lavoz.com.ar/content/los-fachinales-conservan-el-60-del-carbono-de-cordoba
CRAGNOLINO, E. 1997. La unidad doméstica en una investigación de antropología. Ponencia presentada en V Congreso Argentino de Antropología Social. La Plata, Argentina. Disponible: https://www.equiponaya.com. ar/congresos/contenido/laplata/LP5/16.htm

DE GEA, G.S.; PETRYNA, A.M.; MELLANO, A.; BONVILLANI, A.; TURIELLO, P. 2005. El Ganado caprino en la Argentina. Universidad Nacional de Río Cuarto. Argentina. 198 p.

GLASER, B.G.; STRAUSS, A.L. 1967. The Discovery of Grounded Theory. Chicago: Aldine. 345 pp.

HOCSMAN, L.D. Y PREDA, G. 2005. Agriculturización y bovinización. La renovada territorialización capitalista en Córdoba (Argentina). IV Jornadas Interdisciplinarias de Estudios Agrarios y Agroindustriales. CIEA, FCE, UBA. Buenos Aires, Argentina.

HOYOS, L.E.; CINGOLANI, A.M.; ZAK, M.R.; VAIERETTI, M.V.; GORIA, D.E.; CABIDO, M.R. 2013. Deforestation and precipitation patterns in the arid Chaco forests of central Argentina. Applied Vegetation Science, 16 (2): 260-271.

JELIN, E. 1984. Familia y unidad doméstica: mundo público y vida privada. Buenos Aires: Estudios CEDES. 41 pp.

JODELET, D. 1986. La representación social: fenómenos,conceptos y teorías. En: Moscovici S, editor. Psicología Social. Buenos Aires: Paidós. Pp.469-494.

LAND DEGRADATION ASSESSMENT IN DRAYLANDS (LADA). 2004. Informe Preliminar de Evaluación de la Degradación de Tierras en Zonas Áridas. GEF, FIDA. Secretaría de Recursos Naturales. Disponible en: www.fa.org/nr/lada. 
MORELLO, J.; PROTOMASTRO, J.; SANCHOLUZ, L.; BLANCO, C. 1985. Estudio macroecológico de los Llanos de la Rioja. Serie del Cincuentenario de la Administración de Parques Nacionales, 5: 1-53.

NAROTZKY, S. 2004. Antropología Económica. Barcelona: Melusina. 351 pp.

SILVETTI, F.; CÁCERES, D.M.; SOTO, G.; CABROL, D. 2018. Condiciones de persistencia campesina y dinámica del agronegocio en el Norte de la Provincia de Córdoba. Revista FAVE Sección Ciencias Agrarias. 17 (1).

SILVETTI, F. 2010. Estrategias campesinas, construcción social del hábitat y representaciones sociales sobre la provisión de servicios ecosistémicos en el Chaco Árido. Un análisis sociohistórico en el Departamento Pocho (Córdoba, Argentina). [Tesis de Doctorado]. [Córdoba]. Universidad Nacional de Córdoba.

SILVETTI, F. 2011. Una revisión conceptual sobre la relación entre campesinos y servicios ecosistémicos. Cuadernos de desarrollo rural. 8(66): 19-45.

SILVETTI, F. 2012. Trayectoria histórica de la territorialidad ganadera campesina en el oeste de la Provincia de Córdoba (Argentina). Agricultura, Sociedad y Desarrollo. 9 (3): 333-367.

SILVETTI, F.; SOTO, G.; CÁCERES, D.M.; CABROL, D. 2013. ¿Por qué la Legislación no protege a los bosques nativos de Argentina? Conflictos socioambientales y políticas públicas en la provincia de Córdoba. Mundo Agrario. 13 (26).
SILVETTI, F. Y CÁCERES, M.D. 2015. La expansión de monocultivos de exportación en Argentina y Costa Rica. Conflictos socioambientales y lucha campesina por la justicia ambiental. Mundo Agrario. 16 (32). Disponible en: https://www. mundoagrario.unlp.edu.ar/article/view/ MAv16n32a08/6792.

SILVETTI, F.; CÁCERES, D.; SOTO, G.; CABROL, D. 2018. Condiciones de persistencia campesina y dinámica del agronegocio en el Norte de la Provincia de Córdoba. Revista FAVE Sección Ciencias Agrarias, 17 (1): 57-70.

STRAUSS, A.; CORBIN, J. 1990. Basics of qualitative research: Grounded theory procedures and techniques. London: SAGE Publications. $272 \mathrm{pp}$.

VALLES, M. 1997. Técnicas cualitativas de investigación social. Madrid: Editorial Síntesis. $430 \mathrm{pp}$.

WEISS, R. 1995. Learning from strangers. The art and method of qualitative interview studies, New York: The Free Press.

ZAK, M.R. 2008. Patrones espaciales de la vegetación de la provincial de Córdoba. Tesis Doctoral. Facultad de Ciencias Exactas, Físicas y Naturales. [Tesis de Doctorado]. [Córdoba]. Universidad Nacional de Córdoba.

ZAK, M.R.; CABIDO, M.; CÁCERES, D.M.; DÍAZ, S. 2008. What drives accelerate land cover change in central Argentina? Synergistic consequences of climatic, socio-economic and technological factors. Environmental Management. 42 (2): 181-189. 